\title{
Research of Distributed Virtual Agents Based on Queuing Theory
}

\author{
Guangxuan Chen ${ }^{1, \mathrm{a}}$, Huiyun Jin ${ }^{1, \mathrm{~b}}$, Yanhui $\mathrm{Du}^{2, \mathrm{c}^{*}}$, Panke Qin ${ }^{3, \mathrm{~d}}$ and \\ Lei Zhang ${ }^{4, e}$ \\ ${ }^{1}$ Zhejiang Police College, Hangzhou, China \\ ${ }^{2}$ People's Public Security University of China, Beijing, China \\ ${ }^{3}$ College of Computer Science and Technology, Henan Polytechnic \\ University, Jiaozuo, China \\ ${ }^{4}$ The Academy of Logistics, Beijing, China \\ aericcgx@163.com, ${ }^{b}$ jinhuiyun@zjjcxy.cn, ${ }^{c} d y h 6889 @ g m a i l . c o m,{ }^{d}$ qinpan \\ ke@gmail.com, ${ }^{2}$ zxcv123o@163.com \\ * Corresponding author: Yanhui Du (*)
}

\begin{abstract}
.
In order to find a better solution to predict the incoming traffic, grasp the customers' demand in a short time and design a more scientific and reasonable and adjustable schedule for the online customer service center, this paper proposed a new model based on Erlang algorithm. The algorithm can effectively quantify the queuing phenomenon caused by inadequate system capacity in the short time under random environment. Practice shows that the Erlang algorithm based model can enhance the accuracy of the prediction of the agent number and service level, and solve the conflicts between the service level and facility utilization, and finally improve the economic efficiency of the enterprise Keywords: Virtual agents, Erlang algorithm, Customer service,

\section{Introduction}

With the development of internet and the diversification of communication, especially the surprisingly prevalence of cloud computing and big-data, network marketing is becoming increasingly important in the modern business world. As an important means of enhancing the corporate image, customer service is shifting its focus from traditional telephone service to the internet. Online customer
\end{abstract}


service with its convenient web page dialog is opening a new page in the customer service's history.

As for the customers, the convenience of the online customer service can help them eliminate the confusions of the products or services. Communication through online dialog is more straight-forward than telephone and email. In addition, the text dialog is more convenient for customers to capture the emphasis of the consulting information, and to record and store the information for future use. As for the enterprises, online customer service not only can effectively broaden the marketing approach and deepen the relationships with the customers, but also can help the company manage the visitor data and historical data, and thus to better grasp the feedback from the customers and eventually improve the service quality, so as to achieve the visitors into potential customer.

However, the accuracy of the prediction of the incoming traffic, the speed to grasp the customers's demand, the scientificity and rationality of schedule designation and the adjustbality of the schedule are problems to perplex the manangers in the customer service center still. The remainder of this paper is organized as follows: firstly, we proposed a model of the virtual agents according to queuing theory and analysed the algorithm. Then, we introduced the designation of the online customer service center. Finally, we summed up the new algorithm and suggested future study.

\section{Model of Virtual Agents}

It is necessary to predict the income connecting traffic in an upcoming period of time so as to arrange appropriate agents to deal with the customers' demand. The number of requests and average service time are the two main indexes to be predicted according to the historical data. There are many ways to predict the indexes, such as the mean prediction method, moving mean prediction method, exponential smoothing prediction method. However, each method has its own weaknesses and limitations. 
Queuing theory, also known as stochastic service system theory, is a discipline used to research the queuing or congestion phenomenon caused by random factors. Some important quantitative indexes as waiting time, queuing length and busy period can be drawn from the theory through the statistical research on the arrival time of the service clients and service hours. Fig. 1 shows a simple queuing model. Based on queuing theory, we adopted Erlang algorithmthat can effectively solve problems as peak hours affirmation, forecast of manpower needs in working hours [1].

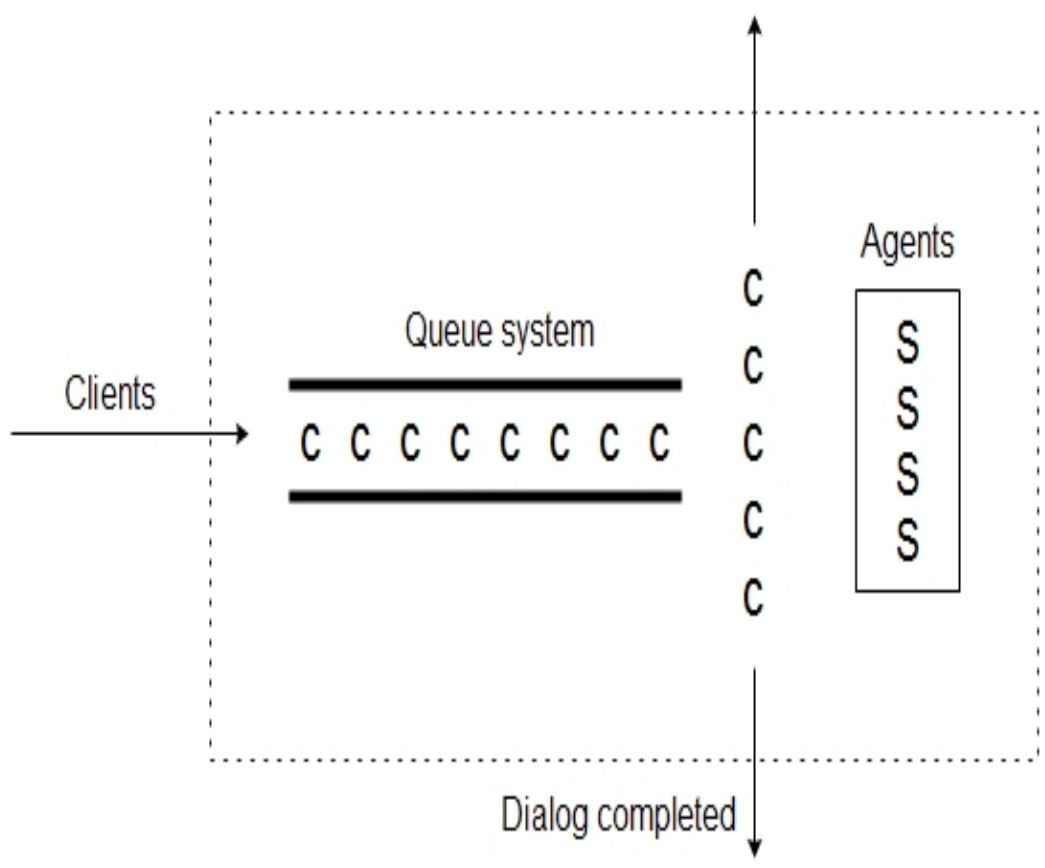

Fig. 1 A simple queuing model

Erlang C model describes the probability of all the $N$ agents are occupied or customers queuing for the service under the conditions that traffic arrival rate, average service time and $N$ parallel agents are given.

A valid Erlang C model should meet following criteria: (1) the features of the customers and the service skills of the agent have no difference in the statistical sense, and do not change with the time; (2) customers have no priority over the service and follow the "first come, first served" rule; (3) the actions as giving up 
service and reconnecting during the arrival process and service process are not considered in the algorithm [2]. The interval of the arrival connecting customers obeys the Poisson distribution with parameter $\lambda$ and the service times of the $N$ agents are independent and obey Exponential distribution. The state transition diagram of Erlang C model is shown in Fig. 2.

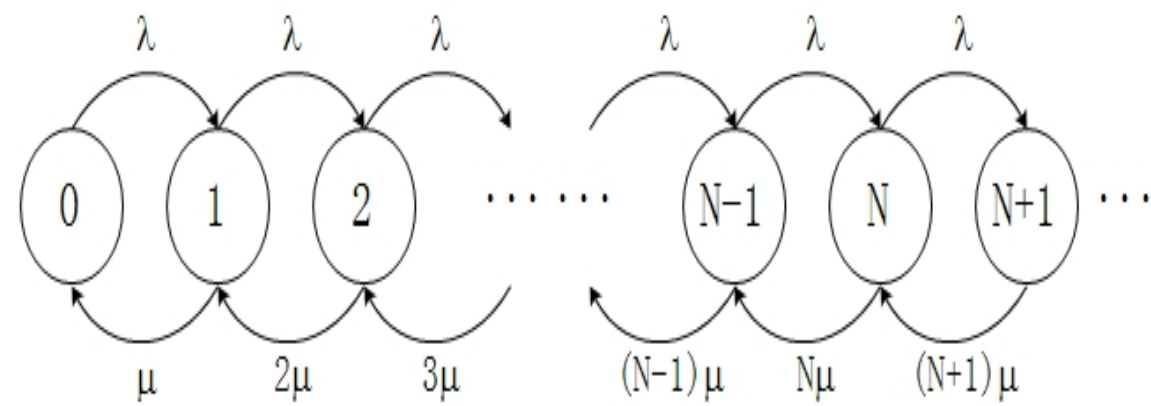

Fig. 2 state transition diagram of Erlang $C$ model

The probability of each connecting customers has to wait can be calculated through Erlang $\mathrm{C}$ equation:

$$
E_{c}(m, u)=\frac{\frac{u^{m}}{m !}}{\frac{u^{m}}{m !}+(1-\rho) \sum_{k=0}^{m-1} \frac{u^{k}}{k !}},
$$

where $m$ refers to the number of agents and $u$ refers to the accessing traffic intensify. The accessing traffic intensity can be drawn through

$$
u=\lambda T_{S},
$$

$$
\rho=\frac{u}{m}
$$


where $\lambda$ refers to the average arrival rate of the connecting customers, $T_{S}$ means the average dialog duration between the customer and the agent and $\rho$ means the occupancy of the agents. Then, we can calculate the average waiting time of the customer through

$$
T_{W}=\frac{E_{c}(\mathrm{~m}, \mathrm{u})\left[T_{\mathrm{S}}\right.}{m \sqsubset(1-\rho)} .
$$

Since the service level is the main index in an online customer service system. It is necessary to set the maximum number of iterations according to the service level when calculating the number of agents. Here, the service level $W(t)$ under the target answer time $t$ can be calculated through

$$
W(t)=1-E_{c}(m, u) \llbracket e^{-(m-u) \square \frac{t}{T_{S}}}
$$

According to the algorithm, we can effectively quantify the queuing phenomenon caused by inadequate system capacity in the short time under random environment. The result can be used as a practical performance management index for the managers in the online customer service center. Meanwhile, combined with employee saturation, time utilization of the agents, load rates of agents and other relative parameters, we found the scheduling of the agents according to this method can effectively enhance the success rate of the connections and improve the quality of the services in the main time period [3]. This can put an end to the phenomenon that the staff unwilling to the night shift which will affect their achievement before.

\section{Structure Designation of the Online Service Center}


With the development of the business and the growth of a variety of customers' needs, the customer service agents based on traditional Web design patterns is increasing imcompetent in dealing with the massive connecting customers. Therefore, it's urgent to change the original fixed agents into virtual agents so as to dynamicly assign and adjust the schedule. Meanwhile, optimizations of the original task scheduling and assigning algorithm are necessary.

The general structure of the distributed virtual agents' work flow in the online customer service center is shown in Fig. 3. The system adopted three-layer mode: presentation layer, business logic layer and data accessing layer.

The presentation layer is mainly comprised of user interaction module and interface controlling module and its main function is to accept the usrs' operation, pass the requests to the service layer and provide application for the clients. The business logic layer located between data accessing layer and presentation layer. It plays a connecting role in the workflow. Here, the layer is a downward dependent weak coupling structure. The lower layer has no knowledge about the upper layer. So, changes on the upper layer have no effect on the lower layer. As for the data accessing layer, it is the caller; and for the presentation layer, it is the callee. The data accessing layer mainly contains the data accessing interface, derived classes that used to implement the interfaces and the enumerations that run under different databases. 


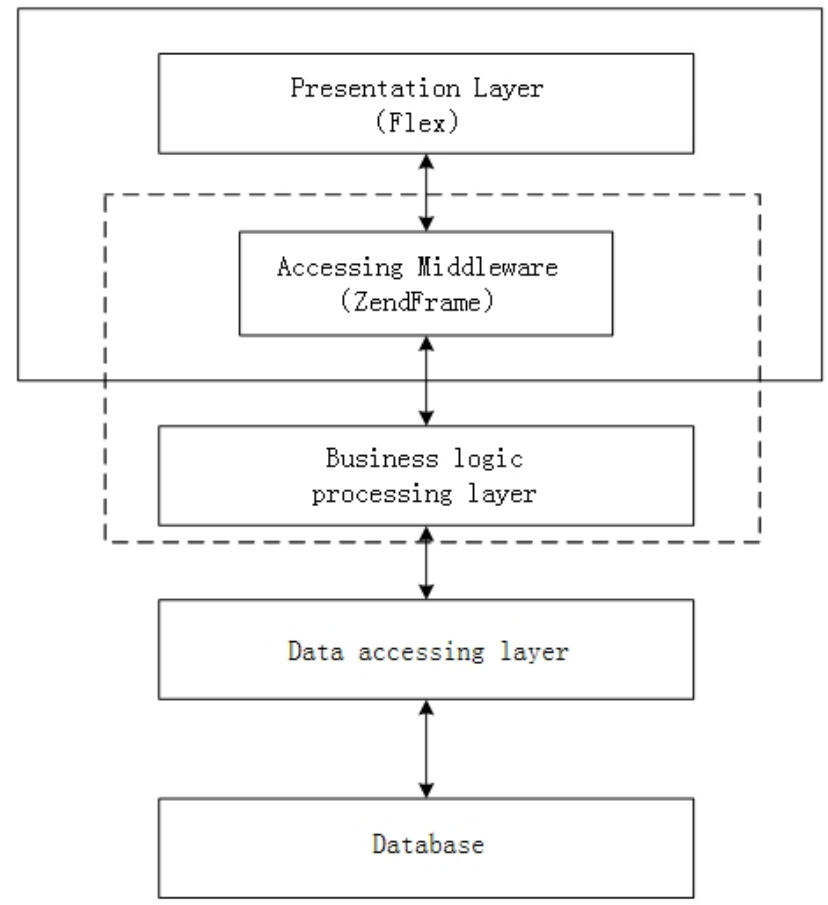

Fig. 3 General structure of the distributed virtual agents' workflow The model can effectively enhance the accuracy of the forecast and can better control the error diffusion of the long period predictions. The prediction of the income connecting can meet the requirements of different granularity forecast. Through state shift analysis, it is possible to very quickly get highly accurate results of customers' demands. Thus, managers in the online service center can reasonably and scientifically set the schedule so as to improve the staff's efficiency and enhance the economic efficiency of the enterprise greatly.

\section{Conclusions}

This paper proposed a new Erlang algorithm based queuing model for the online customer service center. The Erlang $\mathrm{C}$ algorithm describes the probability that an arriving customer will need to queue. It uses the historical data to forecast the number of the agents, peak periods and other relevant parameters. The new model provided a better solution to forecast the incoming traffic and can help the managers to grasp the customer's demands in a short time so as to better design a 
more scientific and reasonable and adjustable schedule for the staff in an enterprise.

\section{References}

[1] Y.J. Q, G.H. X and Q. L: Computer Technology and Development Vol. 20(6) (2010), p. 179

[2] X.J. L, Z.Q. Y, S.J. Z and G.P. Z: Railway Computer Application Vol. 22(10) (2013), p. 17

[3] A.P. W, Y.J. Z and G.Y. Z: Computer Technology and Development Vol. 19(3) (2009), p. 204

[4] Information on http://en.wikipedia.org/wiki/Queueing_theory

[5] Information on http://en.wikipedia.org/wiki/Erlang_(unit)

[6] G.P. Z: Mathematical and Computer Modelling Vol. 37 (37), p. 1287 